\title{
NONLINEAR ROBUST CONTROL OF LARGE-SCALE SYSTEM WITH INPUT SATURATION
}

\author{
Eugenie L. Eremin \\ Informational and Control Systems Department \\ Amur State University \\ Russia \\ ereminel@mail.ru
}

\author{
Larisa V. Nikiforova \\ Informational and Control Systems Department \\ Amur State University \\ Russia \\ chepak@inbox.ru
}

\author{
Evgeniy A. Shelenok \\ Automation and Systems Engineering Department \\ Pacific National University \\ Russia \\ cidshell@mail.ru
}

Article history:

Received 09.03.2021, Accepted 22.06.2021

\begin{abstract}
The article studies control algorithms of multiply connected system for dynamic plants with control saturation and nonlinear cross-connections. The authors of the article offer a decentralized control law based on the hyperstability criterion. They also use this law to constuct the MIMO servo system with input saturation. To illustrate the capability of the proposed decentralized robust control system the authors use an inverted pendulums connected by a spring.
\end{abstract}

\section{Key words}

multiply-connected system, nonlinear robust control, hyperstability criterion, dynamic corrector, input saturation, $L$-dissipativity

\section{Introduction}

The controlling of the nonlinear large-scale systems has a very high theoretical and practical importance. Examples of unstable plants can be found in the various fields of knowledge. In mechanics, for example, it is multiply-connected inverted pendulum (which is multiply connected system itself), or the interconnected system of several inverted pendulums. In technology - operation of a gas turbine engine at low speeds, or control of statically unstable aircraft. The tasks of controlling such plants can also be complicated by the presence of input signals saturations, which can be caused by some design features of technical systems, or by the operating conditions of technical plants.
Methods and techniques of synthesis of the control systems for nonlinear uncertain plants are quite diverse. Noting only some of them ([Monopoli, 1975], [Emelyanov, and Korovin, 1997], [Miroshnik, Nikiforov, and Fradkov, 2000], [Khalil, 2002], [Polyak, and Shcherbakov, 2002], [Bobtsov, 2004], [Bukov, 2006], [Balandin, and Kogan, 2007], [Kolesnikov, 2012], [Eremin, Kvan and Semichevskaya, 2010], [Eremin, Telichenko, and Shelenok, 2010], [Eremin, Telichenko, and Shelenok, 2011]), let us pay attention to the procedures for constructing robust control systems for nonlinear plants under conditions of control signal saturation. The presence of such restrictions affects the performance of control systems and requires the development of special approaches or techniques that ensure their parry ([Feng, Zhang, and Palaniswami, 1991], [Wang, and Sun, 1992], [Zhang, and Evans, 1994], [Yang, Calise, and Craig, 2003], [Takagi, Nishida, and Kobayashi, 2006], [Takagi, Nishida, and Kobayashi, 2007], [Takagi, Oya, Wang, and Kobayashi, 2010], [Takagi, Oya, Wang, and Kobayashi, 2009], [Takagi, Zhuo, Oya, and Wang, 2011], [Takagi, Sato, and Oya, 2011], [Eremin, 2016], [Eremin, and Shelenok, 2016], [Eremin, and Shelenok, 2017]).

This article develops the results of ([Eremin, 2016], [Eremin, 2017]) and expands the area of their application in solving the problem of controlling multiply connected dynamic plants with nonlinear cross-links when the dimensions of vectors of the input and output variables of the plant coincide. 


\section{Preliminaries}

This section is devoted to the mathematical models of the interconnected control system with input saturation that we can present as input-output model or in the statespace forms. Also in this section the problem statement is given.

Let the input-output model of the plant have the following form:

$$
\begin{gathered}
y_{i}(t)=W_{i}(p) U_{i}(t)= \\
=W_{i}(p)\left\{\sigma\left(u_{i}(t)\right)+f_{i}(t)+F_{j}\left(y_{j}(t)\right)\right\} \\
i, j=1,2, \ldots, k ; i \neq j,
\end{gathered}
$$

where $p=d / d t$ is the differentiation operator; $U_{i}(t)$ is some generalized input; $\mathbf{y}^{T}(t)=\left(y_{1}(t), \ldots, y_{k}(t)\right)$ is vector of the output signals; $\mathbf{u}^{T}(t)=\left(u_{1}(t), \ldots, u_{k}(t)\right)$ is vector of the input signals; $\mathbf{f}^{T}(t)=\left(f_{1}(t), \ldots, f_{k}(t)\right)$ is vector of the external disturbances; $F_{j}\left(y_{j}(t)\right)$ are some functions to describe the nonlinear cross-connections; $\sigma\left(u_{i}(t)\right)$ is the nonlinear function of saturation

$$
\sigma\left(u_{i}(t)\right)= \begin{cases}\sigma_{0 i} & u_{i}(t)>\sigma_{0 i} \\ u_{i}(t) & \left|u_{i}(t)\right| \leq \sigma_{0 i} \\ -\sigma_{0 i} & u_{i}(t)<-\sigma_{0 i}\end{cases}
$$

where $\sigma_{0 i}$ are known values.

The plant (1), (2) is supposed to satisfy the following assumptions:

1. Disturbances $f_{i}(t)$ and unknown functions $F_{j}\left(y_{j}(t)\right)$ satisfy the inequalities

$$
\begin{gathered}
\left|f_{i}(t)\right| \leq f_{i}^{*}, f_{i}^{*}=\text { const, } \forall t \geq 0, \\
\left|F_{j}\left(y_{j}(t)\right)\right| \leq F_{j}^{*}, F_{j}^{*}=\text { const }>0, \\
\forall y_{j}(t) \neq 0, F_{j}(0)=0,
\end{gathered}
$$

where $f_{i}^{*}$ and $F_{j}^{*}$ are unknown numbers.

2. Transfer functions of the linear links have the form like

$$
W_{i}(p)=\frac{b_{i}(p)}{a_{i}(p)}, i=1,2, \ldots, k
$$

where $a_{i}(p)=p^{n_{i}}+a_{1 i} p^{\left(n_{i}-1\right)}+\ldots+a_{n_{i} i}$ and $b_{i}(p)=b_{0 i}^{m_{i}}+b_{1 i}^{\left(m_{i}-1\right)}+\ldots+b_{m_{i} i}$ are normalized polynomials with unknown coefficients; $b_{i}(p)$ are Hurwitz polynomials, $b_{0 i}>0 ; a_{i}(p)$ are polynomials with arbitrary roots distribution; $\operatorname{deg} b_{i}(p)=$ $m_{i} \geq 0, \operatorname{deg} a_{i}(p)=n_{i} \geq 1$ are unknown degrees; $\left(\max n_{i}\right),\left(\max m_{i}\right)$ are known limit values; $\rho=\left(\operatorname{deg} a_{i}(p)-\operatorname{deg} b_{i}(p)\right)=\left(n_{i}-m_{i}\right) \geq 1$ are relative orders of the $W_{i}(p) ; \bar{\rho}_{i}=\max \rho_{i}=$ $\left(\max n_{i}-\max m_{i}\right)$ are known values;

3. For the direct measurement only vector $\mathbf{y}(t)$ is available, i. e. the variables $y_{1}(t), \ldots, y_{k}(t)$ (the local subsystems outputs) are available.

Since unknown values $\rho_{i}=$ const belong to a known interval $\bar{\rho}_{i} \geq \rho_{i} \geq 1$ (Assumption 2), it is expedient (see [Eremin, 2018]) to pass the measurable signals $y_{i}(t)$ through the outputs filter-correctors (OFC)

$$
\begin{gathered}
y_{F i}(t)=W_{F i}(p) y_{i}(t)= \\
=\left(\frac{T_{i} p+1}{T_{i}^{*} p+1}\right)^{\bar{\rho}_{i}-1} y_{i}(t), i=1,2, \ldots, k,
\end{gathered}
$$

where $y_{F i}(t)$ and $y_{i}(t)$ are respectively output and input signals; $W_{F i}(p)$ are transfer functions of the $O F C ; T_{i}$ and $T_{i}^{*}$ are time constants. As a result of serial connection of the plant (1) and $O F C$ (6) mathematical model (1) will be transformed like

$$
\begin{gathered}
y_{F i}(t)=W_{F i}(p) W_{i}(p) U_{i}(t)= \\
=\left(\frac{T_{i} p+1}{T_{i}^{*} p+1}\right)^{\bar{\rho}_{i}-1} \frac{b_{i}(p)}{a_{i}(p)} U_{i}(t)= \\
=\frac{\tilde{b}_{i}(p)}{\tilde{a}_{i}(p)} U_{i}(t)=\tilde{W}_{i}(p) U_{i}(t)= \\
=\tilde{W}_{i}(p)\left\{\sigma\left(u_{i}(t)\right)+f_{i}(t)+F_{j}\left(y_{j}(t)\right)\right\}, \\
i=1,2, \ldots, k ; i \neq j,
\end{gathered}
$$

where $\tilde{b}_{i}(p)=b_{i}(p) \cdot\left(T_{i} p+1\right)^{\left(\bar{\rho}_{i}-1\right)}, \tilde{a}_{i}(p)=a_{i}(p)$. $\cdot\left(T_{i}^{*} p+1\right)^{\left(\bar{\rho}_{i}-1\right)}$.

Considering that products $W_{F i}(p) W_{i}(p)$ have the relative degrees $\Delta_{i}=\operatorname{deg} \tilde{a}_{i}-\operatorname{deg} \tilde{b}_{i} \geq 1$, where $\operatorname{deg} \tilde{a}_{i}=$ $n_{i}+\bar{\rho}_{i}-1, \operatorname{deg} \tilde{b}_{i}=m_{i}+\bar{\rho}_{i}-1$ and also taking into account the identities

$$
\begin{gathered}
\tilde{W}_{i}(p)=\frac{b_{i}(p)}{a_{i}(p)} \cdot\left(\frac{T_{i} p+1}{T_{i}^{*} p+1}\right)^{\left(\bar{\rho}_{i}-1\right)}= \\
=\frac{b_{i}(p)}{a_{i}(p)} \cdot \frac{\left(T_{i} p+1\right)^{\left(\bar{\rho}_{i}-1\right)}}{\left(T_{i}^{*} p+1\right)^{\left(\bar{\rho}_{i}-n_{i}+m_{i}\right)}} \times \\
\quad \times \frac{1}{\left(T_{i}^{*} p+1\right)^{\left(n_{i}-m_{i}-1\right)}}= \\
=\frac{\tilde{b}_{i}(p)}{\hat{a}_{i}(p)} \cdot \frac{1}{\left(T_{i}^{*} p+1\right)^{\left(n_{i}-m_{i}-1\right)}}= \\
=\hat{W}_{i}(p) \cdot \frac{1}{\left(T_{i}^{*} p+1\right)^{\left(n_{i}-m_{i}-1\right)}},
\end{gathered}
$$

where $\hat{a}_{i}(p)=a_{i}(p) \cdot\left(T_{i}^{*} p+1\right)^{\bar{\rho}_{i}-n_{i}+m_{i}}, \operatorname{deg} \hat{a}_{i}(p)=$ $=\bar{\rho}_{i}+m_{i}$; and choosing a small values for time constants $T_{i}^{*}$ we can show (by analogy with [Eremin, 2018]) that with the respect to

$$
\frac{1}{\left(T_{i}^{*} p+1\right)^{\left(n_{i}-m_{i}-1\right)}} \cong 1, i=1,2, \ldots, k
$$


it is always permissible to replace model (7) with the following approximate model

$$
\begin{gathered}
y_{F i}(t) \cong \frac{\tilde{b}_{i}(p)}{\hat{a}_{i}(p)} y_{i}(t)=\hat{W}_{i}(p) U_{i}(t)= \\
=\hat{W}_{i}(p)\left\{\sigma\left(u_{i}(t)\right)+f_{i}(t)+F_{j}\left(y_{j}(t)\right)\right\}, \\
i=1,2, \ldots, k ; i \neq j
\end{gathered}
$$

where the relative degrees of $\hat{W}_{i}(p)$ will satisfy the condition $\hat{\rho}_{i}=\operatorname{deg} \hat{a}_{i}(p)-\operatorname{deg} \tilde{b}_{i}(p)=\bar{\rho}_{i}+m_{i}-$ $-\left(\bar{\rho}_{i}+m_{i}-1\right)=1$. It should be noted that degrees of numerators $\left(\bar{\rho}_{i}+m_{i}\right)$ and denominators $\left(\bar{\rho}_{i}+m_{i}-1\right)$ of the transfer functions $\hat{W}_{i}(p)$ are priory unknown.

Let us rewrite model (9) in the state-space form then can be represented as follows:

$$
\begin{gathered}
\frac{d \mathbf{x}_{i}(t)}{d t}=\mathbf{N}_{i} \mathbf{x}_{i}(t)+\mathbf{b}_{i}\left\{\hat{\mathbf{a}}_{i}^{T} \mathbf{x}_{i}(t)+\sigma\left(u_{i}(t)\right)+\right. \\
\left.+f_{i}(t)+F_{i}\left(y_{j}(t)\right)\right\}, y_{F i}(t)=\mathbf{c}_{i}^{T} \mathbf{x}_{i}(t), \\
\mathbf{x}_{i}\left(t_{0}\right)=\mathbf{x}_{i 0}, t \geq t_{0}=0, i, j=1,2, \ldots, k, i \neq j,
\end{gathered}
$$

where $\mathbf{x}_{i}(t)=\left[x_{1 i}(t), \ldots, x_{\left(\bar{\rho}_{i}+m_{i}\right) i}(t)\right]^{T} \in \mathbf{R}^{\left(\bar{\rho}_{i}+m_{i}\right)}$ are state vectors; $\mathbf{N}_{i}$ are nilpotent (upper shift) matrices of $\left(\bar{\rho}_{i}+m_{i}\right) \times\left(\bar{\rho}_{i}+m_{i}\right)$ size; $\mathbf{b}_{i}=$ $=[0, \ldots, 0,1]^{T}$ are vectors of $\left(\bar{\rho}_{i}+m_{i}\right) \times 1$ size; $\quad \hat{\mathbf{a}}_{i}^{T}=\left[\hat{a}_{\left(\bar{\rho}_{i}+m_{i}\right) i}, \ldots, \hat{a}_{2 i}, \hat{a}_{1 i}\right]$ and $\mathbf{c}_{i}^{T}=$ $=\left[\tilde{b}_{\left(\bar{\rho}_{i}+m_{i}-1\right) i}, \ldots, \tilde{b}_{1 i}, \tilde{b}_{0 i}\right]$ are vectors of $\left(\bar{\rho}_{i}+m_{i}\right) \times 1$ and $\left(\bar{\rho}_{i}+m_{i}-1\right) \times 1$ sizes with an appropriate coefficients.

\section{Control Goals}

Let the main control goal is to provide the desired dynamics of the outputs $y_{i}(t)$ that consists in the quality serving to given signals $r_{i}(t)$, i. e. in achieving following conditions at $t \rightarrow \infty$ :

$$
\left|r_{i}(t)-y_{i}(t)\right| \leq \Delta_{0 i}, \Delta_{0 i}=\text { const },
$$

where $\Delta_{0 i}$ are required values.

Wherein the desired dynamics for outputs of the main control loop $y_{F i}$ and also outputs of the $O F C$ are formed with the help of command filter-correctors (CFC) [Eremin, 2018]:

$$
\begin{aligned}
& \hat{r}_{i}(t)=W_{F i}(p) r_{i}(t)= \\
= & \left(\frac{T_{i} p+1}{T_{i}^{*} p+1}\right)^{\left(\bar{\rho}_{i}-1\right)} r_{i}(t),
\end{aligned}
$$

where $\hat{r}_{i}(t)$ are auxiliary command signals.

Then for the plant with $O F C$ (10) that operates in the conditions of functional and parametric uncertainties it is possible to formulate following additional control goal: it is necessary to synthesize an explicit form of the control law so that at measuring only signals $y_{i}(t)$, any initial conditions $\mathbf{x}_{i}(0)$, any disturbances $f_{i}(t)$ (3) and nonlinear cross-connections $F_{i}\left(y_{j}(t)\right)$ (4) at $t \rightarrow \infty$ it will fulfill the following requirements

$$
\left|y_{i}^{*}(t)-y_{F i}(t)\right| \cong\left|\hat{r}_{i}(t)-y_{F i}(t)\right| \leq \hat{\Delta}_{0 i},
$$

where $\hat{\Delta}_{0 i}$ are maximum allowable errors in the tracking mode; $y_{i}^{*}(t)$ are outputs of an implicit reference model $(I R M)$ in the input-output form:

$$
\begin{gathered}
y_{i}^{*}(t)=\frac{1}{\chi_{* i}^{-1} p+1} \hat{r}_{i}(t)=\frac{\chi_{* i}}{p+\chi_{* i}} \hat{r}_{i}(t), \\
\chi_{* i}=\text { const }>0 .
\end{gathered}
$$

It is well known that at $\chi_{* i} \gg 0$ we can rewrite the expression (15) like

$$
y_{i}^{*}(t) \cong \hat{r}_{i}(t) .
$$

Thus, if we provide the additional targets (14), then the main control targets (11) will be fulfilled by virtue of full equivalence of the transfer functions in equations (6) and (12).

Note that for model (10) instead of IRM (15) it is expedient to consider their equivalent analogues [Eremin, 2018]

$$
\begin{aligned}
& y_{i}^{*}(t)=\frac{\chi_{* i} \tilde{b}_{i}(p)}{\left(p+\chi_{* i}\right) \tilde{b}_{i}(p)} \hat{r}_{i}(t) \cong \\
& \cong \frac{\hat{\chi}_{* i} \tilde{b}_{i}(p) \tilde{b}_{\left(\bar{\rho}_{i}+m_{i}-1\right) i}}{\left(p+\chi_{* i}\right) \tilde{b}_{i}(p)} \hat{r}_{i}(t)
\end{aligned}
$$

where $\hat{\chi}_{* i}=\chi_{* i} \tilde{b}_{\left(\bar{\rho}_{i}+m_{i}-1\right) i}^{-1}$, that in the state-space can be represented as follows:

$$
\begin{gathered}
\frac{d \mathbf{x}_{i}^{*}(t)}{d t}=\mathbf{A}_{* i} \mathbf{x}_{i}^{*}(t)+\mathbf{b}_{i} \hat{\chi}_{* i} \hat{r}_{i}(t), \\
y_{i}^{*}(t)=\mathbf{c}_{i}^{T} \mathbf{x}_{i}^{*}(t), \\
\mathbf{x}_{i}^{*}\left(t_{0}\right)=0, i=1,2, \ldots, k ; t \geq t_{0}=0,
\end{gathered}
$$

where $\mathbf{x}_{i}^{*}(t)=\left[x_{1 i}^{*}(t), \ldots, x_{\left(\bar{\rho}_{i}+m_{i}\right) i(t)}^{*}\right]^{T} \in R^{\left(\bar{\rho}_{i}+m_{i}\right)}$; $\mathbf{A}_{* i}=\mathbf{N}_{i}+\mathbf{b}_{i} \hat{\mathbf{a}}_{* i}^{T}=\mathbf{N}_{i}+\mathbf{b}_{i}\left(\hat{\mathbf{a}}_{i}-\chi_{* i} \mathbf{c}_{i}\right)^{T}$ is Hurwitz matrix of $\left(\bar{\rho}_{i}+m_{i}\right) \times\left(\bar{\rho}_{i}+m_{i}\right)$ size; $\chi_{* i}, \hat{\chi}_{* i}=$ const $\gg 0$ are large numbers; $\mathbf{a}_{* i}^{T}=$ $=\left[\hat{a}_{*\left(\bar{\rho}_{i}+m_{i}\right) i}, \ldots, \hat{a}_{* 2 i}, \hat{a}_{* 1 i}\right]=\left(\mathbf{a}_{i}-\chi_{* i} \mathbf{c}_{i}\right)^{T}=$ $=\left[a_{\left(\bar{\rho}_{i}+m_{i}\right) i}-\chi_{* i} \tilde{b}_{\left(\bar{\rho}_{i}+m_{i}-1\right) i}, \ldots, a_{2 i}-\chi_{* i} \tilde{b}_{1 i}, a_{1 i}-\right.$ - $\left.\tilde{b}_{0 i}\right]^{T}$ is a vector which elements are determined by values of polynomial coefficients calculated from $\left(p+\chi_{* i}\right) \tilde{b}_{i}(p) / \tilde{b}_{\left(\bar{\rho}_{i}+m_{i}-1\right) i}$ where coefficient at the highest degree is equal to one. 


\section{Main Results}

In this section with the help of hyperstability criterion the synthesis of the control algorithms for the considered decentralized system is discussed.

Considering the deviation $\mathbf{e}_{i}(t)=\left(\mathbf{x}_{i}^{T}(t)-\mathbf{x}_{i}(t)\right)$ of the state vectors of the IRM (18) and plant with $O F C$ (10), the model of this system can be described by the equations

$$
\begin{gathered}
\frac{d \mathbf{e}_{i}(t)}{d t}=\mathbf{A}_{* i} \mathbf{e}_{i}+\mathbf{b}_{i} \mu_{i}(t), \\
v_{i}(t)=\mathbf{c}_{i}^{T}(t) \mathbf{e}_{i}(t)=y_{i}^{*}(t)-y_{F i}(t)=\hat{r}_{i}(t)-y_{F i}(t), \\
\mu_{i}(t)=\hat{\chi}_{* i} \hat{r}_{i}(t)-\chi_{* i} y_{F i}(t)-u_{i}(t)- \\
-\left(\sigma\left(u_{i}(t)\right)-u(t)\right)-f_{i}(t)-F_{i}\left(y_{j}(t)\right), \\
\mathbf{e}_{i}\left(t_{0}\right)=-\mathbf{x}_{i 0}, t=\geq t_{0}=0, i, j=1,2, \ldots, k, i \neq j,
\end{gathered}
$$

where $v_{i}(t)$ and $\mu_{i}(t)$ are modified outputs and control signals respectively.

It is well known from ([Eremin, 2016], [Eremin, and Shelenok, 2016], [Eremin, and Shelenok, 2017], [Eremin, 2017]) that within the hyperstability criterion, the determination of the explicit form of the components $u_{i}(t)$ of the control system (19) is associated with ensuring the conditions for the existence of two following inequalities:

$$
\begin{aligned}
& \operatorname{Re}\left[\mathbf{c}_{i}^{T}\left(j \omega \mathbf{E}-\mathbf{A}_{*}\right)^{-1} \mathbf{b}_{i}\right]>0, \forall \omega \geq 0, \\
& \eta_{i}(0, t)=-\int_{0}^{t} v_{i}(\varsigma) \mu_{i}(\varsigma) d \varsigma>-\eta_{0 i}^{2} \\
& \eta_{0 i}=\text { const }, \forall t>0, i=1,2, \ldots, k
\end{aligned}
$$

Since the frequency inequality (20) is fulfilled due to

$$
\mathbf{c}_{i}^{T}\left(s \mathbf{E}-\mathbf{A}_{*}\right)^{-1} \mathbf{b}_{i}=\frac{1}{\chi_{* i}^{-1} s+1}
$$

where $s$ is complex variable; then it is necessary to determine the conditions that lead to inequality (21) fulfillment. Let us show the synthesis of the control law (13) as follows

$$
\begin{gathered}
u_{i}(t)=\left(\tilde{h}_{1 i} \hat{r}_{i}^{2}(t)+\tilde{h}_{2 i} y_{F i}^{2}(t)+\tilde{h}_{3 i}\right) \times \\
\times v_{i}(t) \tilde{\delta}_{i}(t), \\
\tau_{i} \frac{d \tilde{\delta}_{i}(t)}{d t}+\tilde{\delta}_{i}(t)=\delta_{i}(t), \tilde{\delta}_{i}(t)(0)=0, \\
\delta_{i}(t)= \begin{cases}1, & \forall\left[\sigma_{i}\left(u_{i}(t)\right)-u_{i}(t)\right] v_{i}(t) \geq 0, \\
\delta_{0 i}, & \forall\left[\sigma_{i}\left(u_{i}(t)\right)-u_{i}(t)\right] v_{i}(t)<0, \\
i=1,2, \ldots, k,\end{cases}
\end{gathered}
$$

where $\tilde{h}_{1 i}, \tilde{h}_{2 i}, \tilde{h}_{3 i}>0$ are some constant values; $\tau_{i}$ are time constants of the dynamic switches $(23) ; \delta_{i}(t)$

are switching functions; $0<\delta_{0 i}<1$ are scaling factor; $\tilde{\delta}_{i}(t)$ are outputs of the dynamic switches (23); it is possible to satisfy the inequality (21). For this purpose we define control signal as $u_{i}(t)=u_{1 i}(t)+u_{2 i}(t)+u_{3 i}(t)$ and rewrite the left side of (21) taking into account (19) like

$$
\begin{gathered}
\eta_{i}(0, t)=\left(\int_{0}^{t} u_{1 i}(\varsigma) v_{i}(\varsigma) d \varsigma-\right. \\
\left.-\hat{\chi}_{* i} \int_{0}^{t} \hat{r}_{i}(\varsigma) v_{i}(\varsigma) d \varsigma\right)+\left(\int_{0}^{t} u_{2 i}(\varsigma) v_{i}(\varsigma) d \varsigma+\right. \\
\left.+\chi_{* i} \int_{0}^{t} y_{F i}(\varsigma) v_{i}(\varsigma) d \varsigma\right)+\left(\int_{0}^{t} u_{3 i}(\varsigma) v_{i}(\varsigma) d \varsigma+\right. \\
\left.+\int_{0}^{t}\left[f_{i}(\varsigma)+F_{i}\left(y_{j}(\varsigma)\right)\right] v_{i}(\varsigma) d \varsigma\right)+ \\
+\int_{0}^{t}\left[\sigma_{i}\left(u_{i}(\varsigma)\right)-u_{i}(\varsigma)\right] v_{i}(\varsigma) d \varsigma= \\
=\sum_{\beta=1}^{3} \eta_{\beta i}(0, t)+\int_{0}^{t}\left[\sigma_{i}\left(u_{i}(\varsigma)\right)-u_{i}(\varsigma)\right] v_{i}(\varsigma) d \varsigma .
\end{gathered}
$$

If we write for the integral $\eta_{1 i}(0, t)$ taking into account constraints (2) and condition $\delta_{i}(t) \geq \delta_{0 i}$ following relation:

$$
\begin{gathered}
\eta_{1 i}(0, t)=\int_{0}^{t} u_{1 i}(\varsigma) v_{i}(\varsigma) d \varsigma-\hat{\chi}_{* i} \int_{0}^{t} \hat{r}_{i}(\varsigma) v_{i}(\varsigma) d \varsigma \pm \\
\pm \delta_{0 i} \hat{\chi}_{* i}^{2} h_{1 i} \int_{0}^{t} \hat{r}_{i}^{2}(\varsigma) v_{i}^{2}(\varsigma) \delta_{i}(\varsigma) d \varsigma \pm \frac{1}{\delta_{0 i}^{2} \hat{\chi}_{* i}^{4} h_{1 i}^{2}} \geq \\
\geq \int_{0}^{t}\left[u_{1 i}(\varsigma)-\delta_{0 i} \hat{\chi}_{* i}^{2} h_{1 i} \hat{r}_{i}^{2}(\varsigma) v_{i}(\varsigma) \delta_{i}(\varsigma)\right] v_{i}(\varsigma) d \varsigma- \\
-\frac{1}{\delta_{0 i}^{2} \hat{\chi}_{* i}^{4} h_{1 i}^{2}},
\end{gathered}
$$

then we can equate the relation in square brackets to zero, and obtain the explicit form of $u_{1 i}$ :

$$
\begin{gathered}
u_{1 i}(t)=\tilde{h}_{1 i} \hat{r}_{i}^{2}(t) v_{i}(t) \delta_{i}(t), \\
\tilde{h}_{1 i}=\delta_{0 i} \hat{\chi}_{* i}^{2} h_{1 i},
\end{gathered}
$$

where $h_{1 i}=$ const $>0$ are arbitrary numbers; and obtain for summand $\eta_{1 i}(0, t)$ following fair estimate

$$
\begin{gathered}
\eta_{1 i}(0, t) \geq-\frac{1}{\delta_{0 i}^{2} \hat{\chi}_{* i}^{4} h_{1 i}^{2}}=-\eta_{01 i}^{2} \\
\eta_{01 i}=\text { const }, \forall t>0 .
\end{gathered}
$$

Let us transform integral $\eta_{2 i}(0, t)$ in the following way: 


$$
\begin{gathered}
\eta_{2 i}(0, t)=\int_{0}^{t} u_{2 i}(\varsigma) v_{i}(\varsigma) d \varsigma+\chi_{* i} \int_{0}^{t} y_{F i}(\varsigma) v_{i}(\varsigma) d \varsigma \pm \\
\pm \delta_{0 i} \chi_{* i}^{2} h_{2 i} \int_{0}^{t} y_{F i}^{2}(\varsigma) v_{i}^{2}(\varsigma) \delta_{i}(\varsigma) d \varsigma \pm \frac{1}{\delta_{0 i}^{2} \chi_{* i}^{4} h_{2 i}^{2}} \geq \\
\geq \int_{0}^{t}\left[u_{2 i}(\varsigma)-\delta_{0 i} \chi_{* i}^{2} h_{21} y_{F i}^{2}(\varsigma) v_{i}(\varsigma) \delta_{i}(\varsigma)\right] v_{i}(\varsigma) d \varsigma- \\
-\frac{1}{\delta_{0 i}^{2} \chi_{* i}^{4} h_{2 i}^{2}} .
\end{gathered}
$$

Then the component $u_{2 i}(t)$ will take the form:

$$
\begin{gathered}
u_{2 i}(t)=\tilde{h}_{2 i} y_{F i}^{2}(t) v_{i}(t) \delta_{i}(t), \\
\tilde{h}_{21}=\delta_{0 i} \chi_{* i}^{2} h_{21},
\end{gathered}
$$

where $h_{21}=$ const $>0$ are arbitrary numbers; and for the integral $\eta_{2 i}(0, t)$ we will have the estimate like:

$$
\begin{gathered}
\eta_{2 i}(0, t) \geq-\frac{1}{\delta_{0 i}^{2} \chi_{* i}^{4} h_{2 i}^{2}}=-\eta_{02 i} \\
\eta_{02 i}=\text { const }, \forall t>0 .
\end{gathered}
$$

We can transform the integral $\eta_{3 i}(0, t)$ as follows:

$$
\begin{gathered}
\eta_{3 i}(0, t)=\int_{0}^{t} u_{3 i}(\varsigma) v_{i}(\varsigma) d \varsigma+ \\
+\int_{0}^{t}\left(f_{i}(\varsigma)+F_{i}\left(y_{j}(\varsigma)\right)\right) v_{i}(\varsigma) d \varsigma \pm \\
\pm \delta_{0 i} h_{3 i} \int_{0}^{t}\left(f_{i}^{*}+F_{i}^{*}\right)^{2} v_{i}^{2}(\varsigma) \delta_{i}(\varsigma) d \varsigma \pm \frac{1}{\delta_{0 i}^{2} h_{3 i}^{2}} \geq \\
\geq \int_{0}^{t}\left[u_{3 i}(\varsigma)-\delta_{0 i} h_{3 i}\left(f_{i}^{*}+F_{i}^{*}\right)^{2} v_{i}(\varsigma) \delta_{i}(\varsigma)\right] \times \\
\times v_{i}(\varsigma) d \varsigma-\frac{1}{\delta_{0 i}^{2} h_{3 i}^{2}} .
\end{gathered}
$$

The component $u_{3 i}(t)$ is synthesized in the following form

$$
\begin{gathered}
u_{3 i}(t)=\tilde{h}_{3 i} v_{i}(t) \delta_{i}(t), \\
\tilde{h}_{3 i}=\delta_{0 i}\left(f_{i}^{*}+F_{i}^{*}\right)^{2} h_{3 i},
\end{gathered}
$$

where $h_{3 i}=$ const $>0$ are arbitrary numbers; then for $\eta_{03 i}(0, t)$ we can obtain following estimate

$$
\begin{gathered}
\eta_{03 i}(0, t) \geq-\frac{1}{\delta_{0 i}^{2} h_{3 i}^{2}}=-\eta_{03 i}, \\
\eta_{03 i}=\text { const }, \forall t>0 .
\end{gathered}
$$

Thus the integral (25) with respect to the estimates (27), (29) and (31) will satisfy the inequality like

$$
\begin{gathered}
\eta_{i}(0, t) \geq \int_{0}^{t}\left(\sigma_{i}\left(u_{i}(\varsigma)\right)-u_{i}(\varsigma)\right) v_{i}(\varsigma) d \varsigma- \\
-\frac{1}{\delta_{0 i}^{2} \hat{\chi}_{* i}^{4} h_{1 i}^{2}}-\frac{1}{\delta_{0 i}^{2} \chi_{* i}^{4} h_{2 i}^{2}}-\frac{1}{\delta_{0 i}^{2} h_{3 i}^{2}} .
\end{gathered}
$$

If now, similarly to [Eremin, 2017], the integral on the right side of this inequality is rewritten as

$$
\begin{aligned}
& \int_{0}^{t}\left(\sigma_{i}\left(u_{i}(\varsigma)\right)-u_{i}(\varsigma)\right) v_{i}(\varsigma) d \varsigma= \\
= & \int_{0}^{t_{*}}\left(\sigma_{i}\left(u_{i}(\varsigma)\right)-u_{i}(\varsigma)\right) v_{i}(\varsigma) d \varsigma+ \\
+ & \int_{t_{*}}^{t}\left(\sigma_{i}\left(u_{i}(\varsigma)\right)-u_{i}(\varsigma)\right) v_{i}(\varsigma) d \varsigma,
\end{aligned}
$$

where $t_{*}$ is a moment in time starting from which in the considered system a condition $\left|u_{i}(t)\right| \leq \sigma_{0 i}$ is obvious, then it can be argued that the following inequalities will be true:

$$
\begin{gathered}
\left|\int_{0}^{t_{*}}\left(\sigma_{i}\left(u_{i}(\varsigma)\right)-u_{i}(\varsigma)\right) v_{i}(\varsigma) d \varsigma\right| \leq \eta_{04 i}^{2}, \\
\eta_{04 i}=\text { const }, \forall t \in\left[0 ; t_{*}\right]
\end{gathered}
$$

due to the boundedness of integrable functions on a finite time interval;

$$
\int_{t_{*}}^{t}\left(\sigma_{i}\left(u_{i}(\varsigma)\right)-u_{i}(\varsigma)\right) v_{i}(\varsigma) d \varsigma=0, \forall t \geq t_{*},
$$

since at $t>t_{*}$ it will be fair $\sigma_{i}\left(u_{i}(t)\right)=u_{i}(t)$.

Therefore, taking into account conditions (33) and (34), relation (32) will satisfy the estimate

$$
\begin{aligned}
\eta_{i}(0, t) \geq & -\frac{1}{\delta_{0 i}^{2} \hat{\chi}_{* i}^{4} h_{1 i}^{2}}-\frac{1}{\delta_{0 i}^{2} \chi_{* i}^{4} h_{2 i}^{2}}-\frac{1}{\delta_{0 i}^{2} h_{3 i}^{2}}-\eta_{04 i}^{2}= \\
& =-\eta_{01 i}^{2}-\eta_{02 i}^{2}-\eta_{03 i}^{2}-\eta_{04 i}^{2}=\eta_{0 i}^{2}
\end{aligned}
$$

that confirms the fulfillment of inequality (21).

Remark. In [Khalil, 2002] it was noted that one of the disadvantages of the strong feedback observers is emergence of peaks in transient processes which often lead to the system instability. In the considered case a similar situation may arise because of fast-acting $O F C$. Indeed the $O F C$ are forcing links at the output of which at small values of time constants $T_{i}^{*}$ and non-zero initial conditions as a rule significant peak emissions are formed.

Therefore, in order to weaken the influence of peaks on the formation of control signals, similar to [Khalil, 
2002], we limit the output of the $O F C$ using nonlinearities of the saturation type and rewrite the control law (22) - (24) as follows

$$
\begin{gathered}
u_{i}(t)=\left(\tilde{h}_{1 i} \hat{r}_{i}^{2}(t)+\tilde{h}_{2 i} \text { sat }\left(y_{F i}(t)\right)^{2}+\tilde{h}_{3 i}\right) \\
\cdot v_{i}(t) \tilde{\delta}_{i}(t), \\
\tau_{i} \frac{d \tilde{\delta}_{i}(t)}{d t}+\tilde{\delta}_{i}(t)=\delta_{i}(t), \tilde{\delta}_{i}(t)(0)=0, \\
\delta_{i}(t)= \begin{cases}1, & \forall\left[\sigma_{i}\left(u_{i}(t)\right)-u_{i}(t)\right] v_{i}(t) \geq 0, \\
\delta_{0 i}, & \forall\left[\sigma_{i}\left(u_{i}(t)\right)-u_{i}(t)\right] v_{i}(t)<0, \\
i=1,2, \ldots, k\end{cases}
\end{gathered}
$$

\section{Simulation}

In this section we apply the obtained decentralized nonlinear robust regulator to control two inverted pendulums connected by a hard spring (Fig. 1).

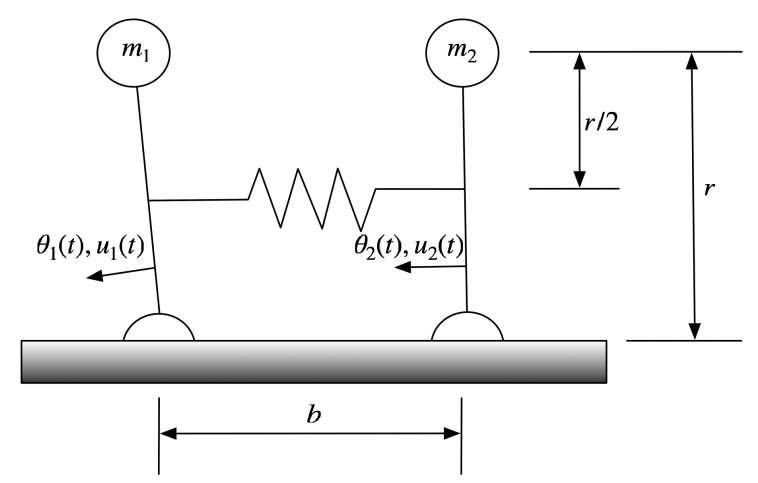

Figure 1. Inverted pendulums

Each pendulum is positioned by a control torque $u_{i}(t), i=1,2$ applied by servomotor at its base. We assume that only angular displacements $\theta_{1}(t)$ and $\theta_{2}(t)$ are available to the direct measurements Nonlinear mathematical model which describes the motion of the such pendulums can be represented as follows [Karimi, and Menhaj, 2010]:

$$
\begin{aligned}
& \left\{\begin{aligned}
\frac{x_{11}(t)}{d t} & =x_{12}(t), \\
\frac{d x_{12}(t)}{d t} & =\left(\frac{m_{1} g r}{j_{1}}-\frac{k r^{2}}{4 j_{1}}\right) \sin \left(x_{11}(t)\right)+ \\
& +\frac{k r}{2 j_{1}}(l-b)+\alpha_{1} \frac{\operatorname{sat}\left(u_{1}(t)\right)}{j_{1}}+ \\
& +\frac{k r^{2}}{4 j_{1}} \sin \left(x_{21}(t)\right),
\end{aligned}\right. \\
& \left\{\begin{aligned}
\frac{x_{21}(t)}{d t} & =x_{22}(t), \\
\frac{d x_{22}(t)}{d t} & =\left(\frac{m_{2} g r}{j_{2}}-\frac{k r^{2}}{4 j_{2}}\right) \sin \left(x_{21}(t)\right)+ \\
& +\frac{k r}{2 j_{2}}(l-b)+\alpha_{2} \frac{\operatorname{sat}\left(u_{2}(t)\right)}{j_{2}}+ \\
& +\frac{k r^{2}}{4 j_{2}} \sin \left(x_{11}(t)\right),
\end{aligned}\right.
\end{aligned}
$$

$$
y_{2}(t)=x_{21}(t), y_{2}(0)=-0.5,
$$

where $x_{11}(t)=\theta_{1}(t)$ and $x_{21}(t)=\theta_{2}(t)$ are vertical angular displacements of the pendulums; $m_{1}=2 \mathrm{~kg}$ and $m_{2}=2.5 \mathrm{~kg}$ are pendulums end masses; $j_{1}=0.5$ $\mathrm{kg} \cdot \mathrm{m}^{2}$ and $j_{2}=0.625 \mathrm{~kg} \cdot \mathrm{m}^{2}$ are moments of inertia; $k=$ $100 \mathrm{~N} / \mathrm{m}$ is the spring constant; $l=0.5 \mathrm{~m}$ is the natural length of the spring; $r=0.5 \mathrm{~m}$ is the pendulum height; $\alpha_{1}=\alpha_{2}=25$ are the control input gains; $g=9.81 \mathrm{~m} / \mathrm{s}^{2}$ is the gravitation acceleration; $b=0.4 \mathrm{~m}$ is the distance between the pendulums binges, $b<1$ indicates that the pendulums repel one another when both are in the upright position.

Functions $\operatorname{sat}(\cdot)=\sigma(\cdot)$ represent nonlinearity of the actuators and set as follows

$$
\begin{aligned}
& \text { sat }\left(u_{1}(t)\right)=\sigma_{1}\left(u_{1}(t)\right)= \\
& = \begin{cases}17, & u_{1}(t)>17, \\
u_{1}(t), & \left|u_{1}(t)\right| \leq 17, \\
-17, & u_{1}(t)<-17 ;\end{cases} \\
& \text { sat }\left(u_{2}(t)\right)=\sigma_{2}\left(u_{2}(t)\right)= \\
& = \begin{cases}15, & u_{2}(t)>15, \\
u_{2}(t), & \left|u_{2}(t)\right| \leq 15, \\
-15, & u_{2}(t)<-15 .\end{cases}
\end{aligned}
$$

We define dynamics of the $O F C$ and $C F C$ like

$$
\begin{aligned}
y_{F i} & =\frac{0.05 p+1}{0.001 p+1} y_{i}(t), \\
\hat{r}_{i}(t) & =\frac{0.05 p+1}{0.001 p+1} r_{i}(t) .
\end{aligned}
$$

At first, let us attempt to regulate the angular position of each pendulum to zero. In this case we define command signals of the subsystems as $r_{1}(t)=r_{2}(t)=r *=$ 0 . In the course of simulation parameters of the robust regulator were chosen with following values:

$$
\begin{gathered}
\tilde{h}_{1 i}=500, \tilde{h}_{2 i}=300, \tilde{h}_{3 i}=300 \\
\delta_{0 i}=0.2, \tau_{i}=0.2 \\
\operatorname{sat}\left(y_{=} F i(t)\right)= \begin{cases}10, & y_{F i}(t)>10 \\
y_{F i}(t), & \left|y_{F i}(t)\right| \leq 10 \\
-10, & y_{F i}(t)<-10\end{cases} \\
i=1,2 .
\end{gathered}
$$

In Fig. 2 and Fig. 3 the pendulums positions and the signals $r^{*}$ are depicted. 


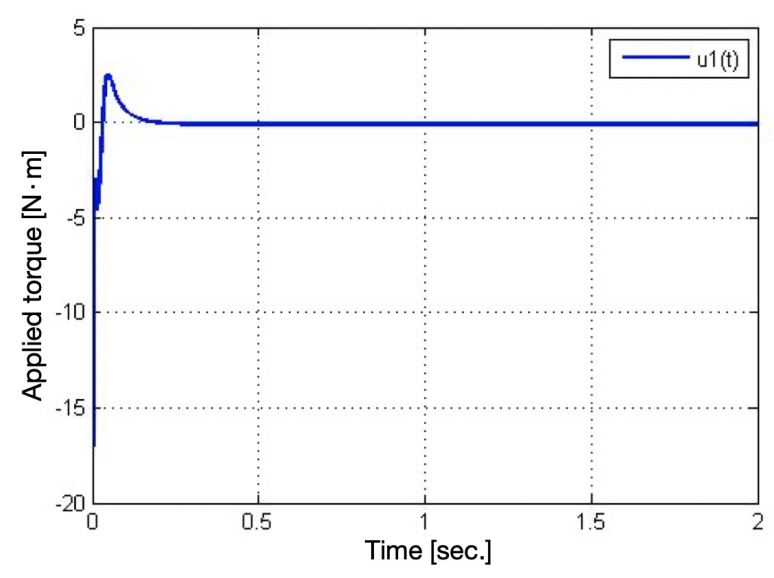

Figure 4. Control torque $u_{1}(t)$ of the first pendulum

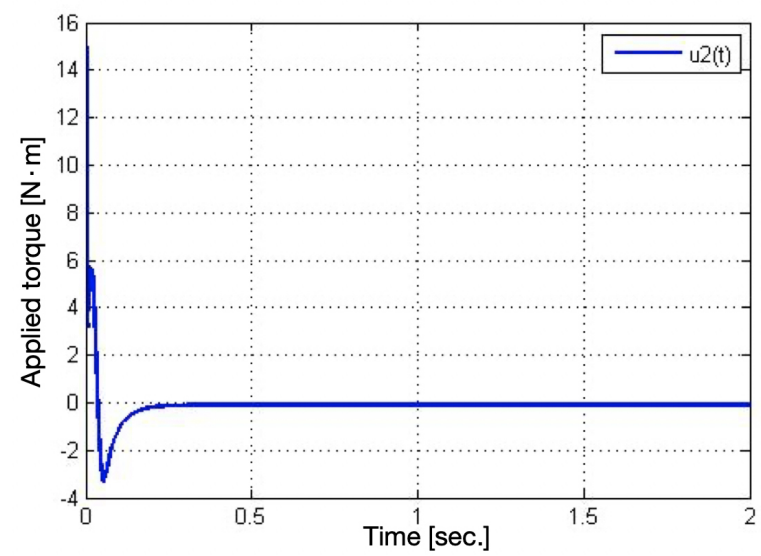

Figure 5. Control torque $u_{2}(t)$ of the fpendulum

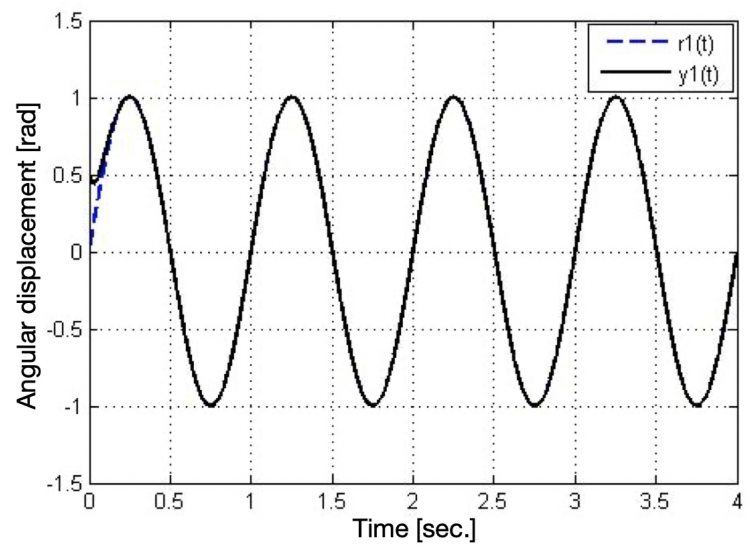

Figure 6. Desired trajectory $r_{1}(t)$ and the first pendulum actual position $y_{1}(t)$

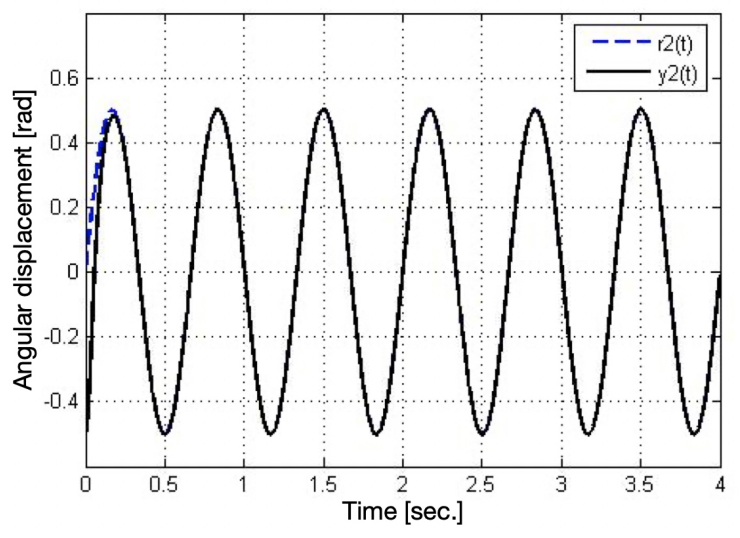

Figure 7. Desired trajectory $r_{2}(t)$ and the first pendulum actual position $y_{2}(t)$

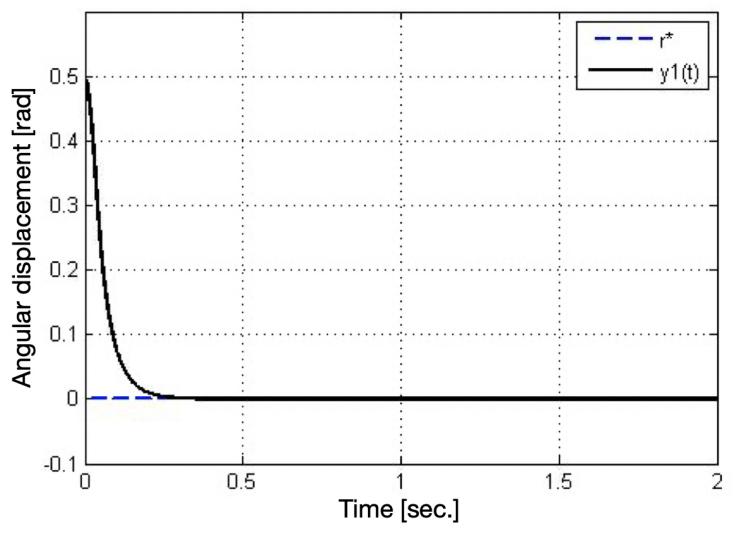

Figure 2. Desired trajectory $r^{*}$ and the first pendulum actual position $y_{1}(t)$

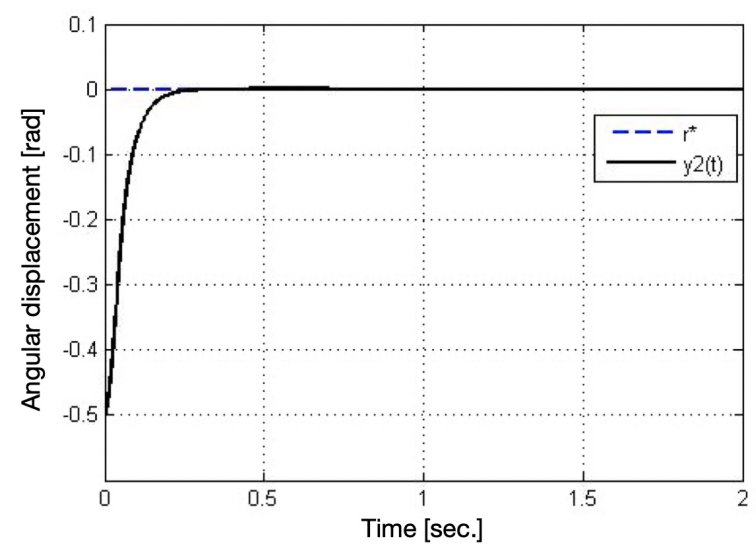

Figure 3. Desired trajectory $r^{*}$ and the second pendulum actual position $y_{2}(t)$

In Fig. 4. and Fig. 5 the dynamics of control torques applied at pendulums actuators are depicted.

At second, we set the desired trajectory of each pendulums as follows

$$
\begin{aligned}
& r_{1}(t)=\sin (6.28 t), \\
& r_{2}(t)=0.5 \sin (9.42 t),
\end{aligned}
$$




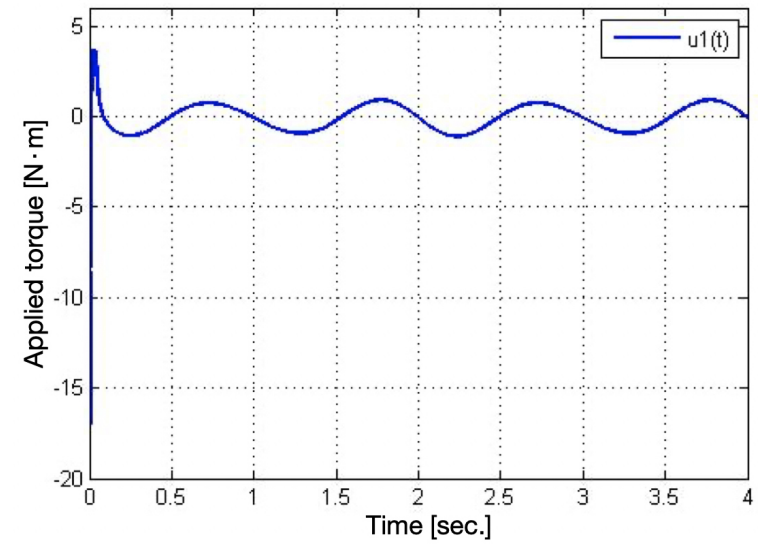

Figure 8. Control torque $u_{1}(t)$ of the first pendulum

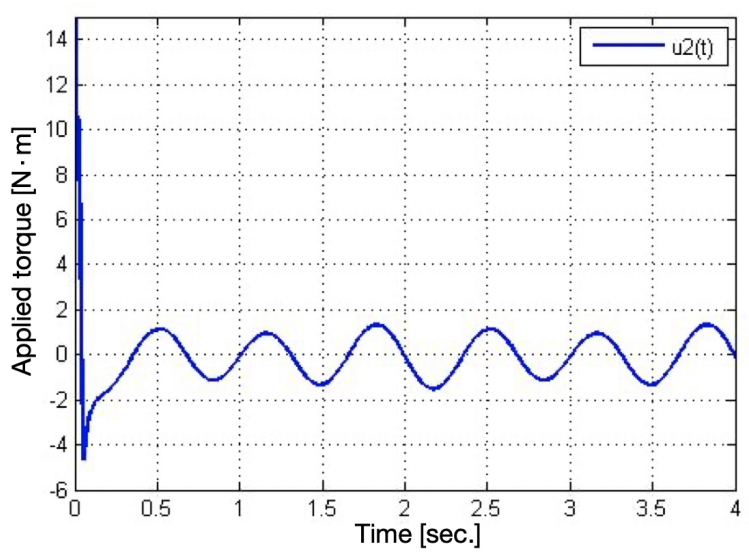

Figure 9. Control torque $u_{2}(t)$ of the first pendulum

ulator parameters (41). The results of the system simulation are depicted in Fig. 6 - 9 .

From the presented results, one can see that the proposed control algorithm (36) - (38), (41) ensures high quality of the system for various operating modes (stabilization of the pendulums position and the movement of the pendulums along given trajectories) without reconfiguring the parameters of the regulator (41). In both cases, due to the formation of control torques, a good enough performance of the control system is ensured (control errors in both local control loops are practically equals zero).

\section{Conclusion}

With the help of hyperstability criterion, the solution of the problem of decentralized control for class of multiply connected dynamic plant which contains nonlinear cross-links is considered. Using the high-speed dynamic correctors and implicit reference model the robust control law is synthesized. This robust regulator, as it is shown at the stage of simulation in relation to the control of two inverted pendulums, makes it possible to achieve a given control goal with a sufficiently high quality.
The obtained results may be useful to construct the control system for mechanical systems such as control systems for robotic manipulators. Also an approach considered in paper can be used for developing the control system for plants which contain state delay.

\section{Acknowledgements}

The work was supported by the Russian Foundation for Basic Research (projects 20-08-00712).

\section{References}

K.J. Astrom and T. Hagglund. Advanced PID-control. ISA (The Instrumentation, Systems and Automation Society), 2006.

D.V. Balandin and M.M. Kogan. Synthesis of control laws based on matrix inequalities. M.: Nauka, 2007.

A.A. Bobtsov. Synthesis of Control Law for Stabilization of a Nonlinear System Based on Measurements of Output. Journal of Computer and Systems Sciences International, 43(3):363-368, 2004.

V.N. Bukov. Nesting systems. An analytical approach to the analysis and synthesis of matrix systems. Kaluga: Publishing house of scientific literature N.F. Bochkareva, 2006.

S.V. Emelyanov and S.K. Korovin. New types of feedback: control under uncertainty. M.: Nauka. Fizmatlit, 1997.

E.L. Eremin. A Modified Adaptive Adaptive System for Controlling the Single-Channel Plant with Inlet Saturation. Information Science and Control Systems, 4(50): 94-102, 2016.

E.L. Eremin. Combined System with an Implicit Standard for a Class of uncertain a Priory Single-Channel Non-Affine Control Plants on the Set of Operation States. Information Science and Control Systems, 3(57):93-103, 2018.

E.L. Eremin. Modification of Regulator of Robust Control System for Nonlinear SISO-Plant with Input Constraints. Information Science and Control Systems, 1(51):64-74, 2017.

E.L. Eremin, N.V. Kvan and N.P. Semichevskaya. Robust Control of Nonlinear Object with High-Speed Obvious-Implicit Standard Model. Mechatronics, automation, control, 5:2-6, 2010.

E.L. Eremin, and E.A. Shelenok. Adaptive control of a single-channel object in a circuit with dynamic correctors and taking into account the saturation of the control signal. Information Science and Control Systems, 4(50):94-102, 2010.

E.L. Eremin, and E.A. Shelenok. The system of decentralized adaptive control of a robotic manipulator with input saturation. Sensors and systems, 1(210):20-27, 2017.

E.L. Eremin, D.A. Telichenko and E.A. Shelenok. Cyclical Mode in Robust Control System for Barrett Manipulator. Bulletin PNU, 3:23-32, 2010. 
E.L. Eremin, D.A. Telichenko and E.A. Shelenok. Periodic modes in decentralized adaptive and robust control schemes. Bulletin of the Ryazan State Radio Engineering University, 35:108-116, 2011.

G. Feng, C. Zhang, and M. Palaniswami. Stability analysis of input constrained continuous time indirect adaptive control. Systems and Control Letters, 17(3):209$215,1991$.

B. Karimi, and M.B. Menhaj. Non-affine nonlinear adaptive control of decentralized large-scale systems using neural networks. Information Sciences, 180: 3335-3347, 2010.

H.K. Khalil. Nonlinear Systems. 3rd ed., Upper Saddle River, N.J.: Prentice Hall, 2002.

A.A. Kolesnikov. Synergetic methods for managing complex systems: the theory of system synthesis. Ed. 2, M.: Librokom, 2012.

I.V. Miroshnik, V.O. Nikiforov, and A.L. Fradkov. Nonlinear and adaptive control of complex dynamic plants. SPb.: Nauka, 2000.

R.V. Monopoli. Adaptive control for system with hard saturation. Proc. of IEEE 14th Conf. on Decision and Control, 841-842, 1975.

B.T. Polyak, and P.S. Shcherbakov. Robust stability and control. M.: Nauka, 2002.

N. Takagi, T. Nishida, and T. Kobayashi. Design of Adaptive Control Systems with Input Saturation. ISICE-ICASE International Joint Conference, 984987, 2006.

N. Takagi, T. Nishida, and T. Kobayashi. Design of adaptive control systems for unstable plants in the presence of input saturation. IIEEE SICE Annual Conference, 454-457, 2007.

N. Takagi, M. Oya, Q. Wang, and T. Kobayashi. Adaptive control of systems with input saturation: A scheme using output derivatives of order up to relative degree. IEEE International Symposium on Computational Intelligence in Robotics and Automation (CIRA), 415420, 2009.

N. Takagi, M. Oya, Q. Wang, and T. Kobayashi. Adaptive control scheme achieving smooth control input in the presence of input saturation. Int. J. Advanced Mechatronic Systems, 2(4):225-235, 2010.

N. Takagi, K. Sato, M. A. Oya. A modified adaptive control scheme in the presence of input saturation. Int. J. Advanced Mechatronic Systems, 3(3):168-180, 2011.

N. Takagi, J. Zhuo, M. Oya, and Q. Wang. Modification of an adaptive controller for systems with input saturation and available output derivatives up to the order of relative degree. IEEE International Symposium on $\mathrm{Ad}$ vanced Control of Industrial Processes (ADCONIP), 114-119, 2011.

H. Wang, and J. Sun. Modified model reference adaptive control with saturated inputs. Proc. Of IEEE 31st Conf. on Decision and Control, 3255-3256, 1992.

B.-J. Yang, A.J. Calise, and J.I. Craig. Adaptive output feedback control with input saturation. Proc. of 2003 American Control Conference, 1572-1577, 2003.

C. Zhang, and R.J. Evans. Continuous direct adaptive control with saturation input constraint. IEEE Tans. on Automatic Control, 39(8):1718-1722, 1994. 